\title{
Monitoring the Long-Distance Transport of Fusarium graminearum from Field-Scale Sources of Inoculum
}

Aaron J. Prussin, II, Department of Plant Pathology, Physiology, and Weed Science, Virginia Tech, Blacksburg, VA 24061-0390; Qing Li and Rimy Malla, Department of Statistics, Virginia Tech, Blacksburg, VA 24061-0219; Shane D. Ross, Department of Engineering Science and Mechanics, Virginia Tech, Blacksburg, VA 24061-0219; and David G. Schmale, III, Department of Plant Pathology, Physiology, and Weed Science, Virginia Tech, Blacksburg, VA 24061-0390

\begin{abstract}
Prussin, A. J., II, Li, Q., Malla, R., Ross, S. D., and Schmale, D. G., III. 2014. Monitoring the long-distance transport of Fusarium graminearum from field-scale sources of inoculum. Plant Dis. 98:504-511.

The fungus Fusarium graminearum causes Fusarium head blight (FHB) of wheat. Little is known about dispersal of the fungus from field-scale sources of inoculum. We monitored the movement of a clonal isolate of $F$. graminearum from a $3,716 \mathrm{~m}^{2}(0.372 \mathrm{ha})$ source of inoculum over two field seasons. Ground-based collection devices were placed at distances of 0 (in the source), 100, 250, 500, 750, and 1,000 $\mathrm{m}$ from the center of the clonal sources of inoculum. Three polymorphic microsatellites were used to identify the released clone from 1,027 isolates (790 in 2011 and 237 in 2012) of the fungus. Results demon-

strated that the recovery of the released clone decreased at greater distances from the source. The majority $(87 \%, 152 / 175$ in $2011 ; 77 \%$, $74 / 96$ in 2012) of the released clone was recaptured during the night (1900 to 0700). The released clone was recovered up to $750 \mathrm{~m}$ from the source. Recovery of the released clone followed a logistic regression model and was significant $(P<0.041$ for all slope term scenarios $)$ as a function of distance from the source of inoculum. This work offers a means to experimentally determine the dispersal kernel of a plant pathogen, and could be integrated into management strategies for FHB.
\end{abstract}

Many plant pathogens use the atmosphere to move over long distances $(1,2)$, including Peronospora tabacina, causal agent of tobacco blue mold (4), Phakopsora pachyrhizi, causal agent of Asian soybean rust $(4,21)$, and Puccinia graminis f. sp. tritici, causal agent of wheat stem rust (40). The atmospheric transport of plant pathogens is broadly characterized by the aerobiological processes of liberation, horizontal transport, and deposition (18). A detailed understanding of these processes is a prerequisite to the development of successful plant disease management strategies.

Another important plant pathogen that utilizes the atmosphere for long-distance transport is Fusarium graminearum (synonym Gibberella zeae) (24,37). F. graminearum is a fungal plant pathogen that causes Fusarium head blight (FHB) of wheat and barley, which has resulted in more than $\$ 3$ billion in crop losses in the United States over the past two decades $(25,29,33)$. The fungus produces a mycotoxin known as deoxynivalenol (DON) that may contaminate food and feed and threaten the health of humans and livestock $(38,41)$.

The disease cycle of $F$. graminearum has been well studied (33). The fungus overwinters in crop debris (e.g., residues of wheat, corn, and barley) from previous growing seasons (12). In the spring, perithecia develop on crop residues and forcibly discharge ascospores millimeter-scale distances $(11,32,42,43)$. These ascospores have the potential to move over long distances by wind $(24,36)$, and may infect fields in neighboring regions. Dill-Macky and Jones (10) found that FHB incidence and severity were highest when wheat followed a susceptible host (corn) and least when wheat followed a nonsusceptible host (soybeans). Reduced-till or no-till systems are expected to result in increased disease and my-

Corresponding author: David G. Schmale, III, E-mail: dschmale @ vt.edu

* The $\boldsymbol{e}$-Xtra logo stands for "electronic extra" and indicates that two supplementary figures are available in the online edition.

Accepted for publication 2 October 2013.

http://dx.doi.org/10.1094/PDIS-06-13-0664-RE

(C) 2014 The American Phytopathological Society cotoxin (DON) levels, since local (within-field) residues of corn and small grains may serve as sources of inoculum $(10,19,20)$. Such sources of inoculum also have the potential to impact neighboring fields, and research is needed to unambiguously track the long-distance movement $(>100 \mathrm{~m})$ of the fungus from a known source (e.g., an infected field) to a final destination (e.g., a susceptible crop).

Recently, there have been advances in release-recovery experiments of $F$. graminearum over short distances $(<30 \mathrm{~m})$ using a genotyping method known as amplified fragment length polymorphism (AFLP) $(19,20)$. In these studies, small amounts of inoculum ( 45 to $410 \mathrm{~g}$ ) were released from $0.55 \mathrm{~m}^{2}$ plots, and diseased wheat heads were collected short distances $(<30 \mathrm{~m})$ from the source of inoculum and analyzed for the released clone of $F$. graminearum. Although these studies were among the first in this pathosystem to track the fungus from known inoculum sources, the size of the inoculum sources and the scale of the experiments limited collections of released clone to within distances of $30 \mathrm{~m}$ from the source. Fernando et al. (14) used an isolate of $F$. graminearum exhibiting a yellow phenotype on standard culture medium to help distinguish the released clone from natural (background) populations of the fungus. Both of these techniques (AFLPs and the release of a yellow phenotype) have some limitations. AFLPs require digestion and ligation reactions with different enzymes followed by multiple polymerase chain reactions (PCRs), and the physical location of and selection on AFLP markers is often unknown. The release and recapture of a yellow phenotype of $F$. graminearum is also limited since yellow phenotypes of the fungus may be present in natural populations of the fungus. An alternative strategy to identify a specific fungal individual from a background population is to use a series of microsatellite markers-short conserved sequences of DNA scattered across the genome (46). The use of microsatellites to identify a released clone of $F$. graminearum within a heterogeneous natural population provides an alternative method to track the long-distance movement of $F$. graminearum from a known inoculum source.

The specific objective of this study was to unambiguously monitor the long-distance transport (up to $1,000 \mathrm{~m}$ ) of $F$. graminearum from a field-scale source of clonal inoculum. Although Schmale et al. (37) speculated that spores of $F$. graminearum may be trans- 
ported tens to hundreds of kilometers in the atmosphere, detailed studies on the long-distance transport (on the scale of $\sim 1,000 \mathrm{~m}$ ) of $F$. graminearum from known sources of inoculum are lacking. We hypothesized that the recovery of the released clone would decrease at increasing distances downwind from a large area source of inoculum (31).

\section{Materials and Methods}

Experimental fields. Field studies were conducted at the Kentland Farm in Blacksburg, VA from 26 April to 25 May 2011 and 9 April to 14 May 2012. The Kentland Farm is composed of about 810 hectares of farmland. Two hectares of winter wheat (untreated Southern States variety SS560) were planted in October 2010 for the 2011 field experiment and October 2011 for the 2012 field experiment. The winter wheat fields were not treated with any fungicides. Approximately 1 month prior to the start of the field experiments, nitrogen was applied at a rate of $56 \mathrm{~kg} / \mathrm{ha}$ to the wheat plots.

Preparation of clonal inoculum sources. Mature, green corn stalks were collected in August 2010 and 2011 from corn fields at the Kentland Farm and dried in a greenhouse for 6 months. The dried corn stalks were cut into $\sim 15-\mathrm{cm}$-long pieces and placed into 50 individual 18.93-liter steel buckets (Global Industrial, Port Washington, NY). Each of 50 buckets was filled approximately $2 / 3$ full with cut corn stalks, sealed with a lid, and autoclaved for 120 min. After the initial autoclaving step, the corn stalks were soaked in deionized water overnight, the water was then removed, and the corn stalks were autoclaved again for $120 \mathrm{~min}$. The autoclaved corn stalks were then inoculated with colonized agar pieces of $F$. graminearum isolate Fg_Va_GPS13N4_3ADON (hereafter referred to as FGVA4) from five 100-mm-diameter petri dishes that had been cultured on 1/4-strength PDA for 12 days. The buckets containing the inoculated corn stalks were stored at ambient room temperature for approximately 10 weeks to allow the fungus to colonize the corn stalks.

A plot area of 0.372 ha of wheat was subdivided into 100 square plots $(10$ rows of 10 plots, $6.096 \times 6.096 \mathrm{~m}$ ). Field inoculations were performed on 2 May 2011 (season 1) and 16 April 2012 (season 2) by spreading corn stalks from each of the 50 buckets into 50 of the subplots in a checkerboard pattern (stalks from one bucket were used for each of the subplots). The field inoculation dates corresponded with when the wheat heads began to boot (Zadok growth stage 10) (16). Perithecia were first observed within 10 days of the corn stalks being placed in the field.

Collection of the released clone at different distances from the source. Petri plates $(100 \mathrm{~mm}$ in diameter; surface area $=78.5$ $\mathrm{cm}^{2}$ ) containing a Fusarium selective medium (FSM) were placed on the top of a 1-m wooden stake to collect viable spores of Fusarium at distances of 0 (in the source), 100, 250, and $500 \mathrm{~m}$ from the center of the inoculated field in 2011, and 0 (in the source), 100, 250, 500, 750, and 1,000 $\mathrm{m}$ from the center of the inoculated field in 2012. The FSM was prepared as previously described (36). In 2011, three sampling devices were placed in the inoculum source, 12 were placed in a circle $100 \mathrm{~m}$ from the center of the source, and five were placed at 250 and $500 \mathrm{~m}$ from the source center in the prevailing downwind direction (Supplementary Figure S1). Three additional sampling devices were placed in the prevailing upwind direction (two were placed $250 \mathrm{~m}$ from the source center and one was placed $500 \mathrm{~m}$ from the source center). In 2012, three sampling devices were placed in the field scale source, 12 were placed in a circle $100 \mathrm{~m}$ from the center of the source, five were placed 250 and $500 \mathrm{~m}$ from the source center in the prevailing downwind direction, and six were placed 750 and $1,000 \mathrm{~m}$ from the source center in the prevailing downwind direction. Three additional sampling devices were placed in the prevailing upwind direction (two were placed $250 \mathrm{~m}$ from the source center and one was place $500 \mathrm{~m}$ from the source center). Wind data were collected from the Virginia Agricultural Experimental Station Mesonet weather station located at Kentland Farm, approximately $250 \mathrm{~m}$ northwest from the center of the source of inoculum in 2011, and approximately $350 \mathrm{~m}$ northwest from the center of the source of inoculum in 2012. Wind speed and direction was recorded at 15-min intervals and used to illustrate the fraction of time the wind was coming from 45 degree sectors during the day, night, and day and night combined from 26 April to 26 May for 2009, 2010, and 2011 and 9 April to 14 May for 2012 (Supplementary Figure S2). Knowledge of the prevailing wind direction and the farm topography (hills, forest, river, etc.) guided the placement of the sampling devices.

In 2011, samples were collected continuously over four time intervals each day: 0700 to 1100,1100 to 1500,1500 to 1900 , and 1900 to 0700 . Sampling was also conducted immediately prior to the release of the clone into the field from 26 April to 2 May 2011 (the field was inoculated 2 May 2011) to assess the presence of the released clone in background populations of $F$. graminearum. Field sampling for the released clone started immediately following the observation of perithecia on the inoculated corn stalks. Field samples were collected for 14 consecutive days (12 May through 25 May 2011).

In 2012, samples were collected continuously over two time intervals each day: 0700 to 1100 and 1900 to 0700 . Sampling was also conducted prior to the release of the clone into the field from 9 April to 12 April 2012 (the field was inoculated 16 April 2012) to assess the presence of the released clone in background populations of $F$. graminearum. Field sampling for the released clone started immediately following the observation of perithecia on the inoculated corn stalks. Field samples were collected for 19 consecutive days (26 April through 14 May 2012).

Fungal isolation and DNA extraction. After each sampling period, exposed petri plates were immediately removed from the field, covered, and placed in small plastic boxes for transport to the laboratory. The plates were incubated for 7 to 10 days in the laboratory at ambient room temperature, and the number of Fusarium colonies (distinct white, fluffy colonies approximately $15 \mathrm{~mm}$ in diameter) collected at each location during each sampling period was recorded. In 2011, up to five Fusarium colonies from each plate were randomly selected and subcultured to petri plates containing 1/4-strength PDA. In 2012, the number of Fusarium samples further subcultured and studied was increased to 10 colonies per plate. Colonies producing red, pink, or yellow mycelia characteristic of $F$. graminearum and containing only macroconidia on $1 / 4-$ strength PDA were purified by transferring a single spore to an additional plate. Single-spored cultures were placed in $20 \%$ glycerol and stored at $-80^{\circ} \mathrm{C}$. Single-spored isolates identified as $F$.

Table 1. Microsatellite primers used for the identification of released clone FGVA4 of Fusarium graminearum

\begin{tabular}{|c|c|c|c|c|c|c|}
\hline Name & Sequence, $5^{\prime}-3^{\prime}$ & $\begin{array}{l}\text { Allelic size } \\
\text { range }(b p)^{\mathrm{a}}\end{array}$ & $\begin{array}{l}\text { Number of } \\
\text { alleles }\end{array}$ & $\begin{array}{l}\text { Released isolate (FGVA4) } \\
\text { allelic size (bp) }\end{array}$ & $\begin{array}{l}\mathbf{T}_{\text {anneal }} \\
\left({ }^{\circ} \mathbf{C}\right)\end{array}$ & $\begin{array}{c}\text { Fluorescen } \\
\text { label }\end{array}$ \\
\hline FusSSR22 & $\begin{array}{l}{ }^{\mathrm{f}} \text { GAGGGCGATGGTTGAAGTGTAC } \\
{ }^{\mathrm{r}} \text { TGGGCATGAAACAAGAGAGAGAC }\end{array}$ & $200-212$ & 4 & 200 & 65 & $\mathrm{Ned}$ \\
\hline FusSSR 23 & $\begin{array}{l}{ }^{\mathrm{f}} \text { GTTGACACAGAAGAATGGCAGG } \\
{ }^{\mathrm{r} C G C T A G G T A C A A A T T G C T G G G ~}\end{array}$ & $165-221$ & 19 & 167 & 65 & Ned \\
\hline FusSSR27 & $\begin{array}{l}{ }^{\mathrm{f} T C A C C A A A A G T C T C C T C A G T C A A C} \\
{ }^{\mathrm{r}} \text { GTGGTCTCCGTAAACGAGCC }\end{array}$ & $157-205$ & 12 & 183 & 65 & Fam \\
\hline
\end{tabular}

a Reported by Vogelgsang et al. (46) based on 33 isolates, five of which were from the United States. 
graminearum were grown in $100 \mathrm{ml}$ of $1 / 4$-strength $\mathrm{PD}$ broth on a shaker at $100 \mathrm{rpm}$ for 5 to 7 days at ambient room temperature. Harvesting of mycelia and extraction of DNA were conducted following previously published methods $(19,20)$.

Identification of the released clone from field collections. Three microsatellites (FusSSR22, FusSSR23, and FusSSR27) were used to genotype the singled-spored isolates of $F$. graminearum from the field collections (Table 1 [46]). The forward primers were labeled with fluorescent dyes (FusSSR22 was labeled with Ned, FusSSR23 was labeled with Ned, and FusSSR27 was labeled with Fam), and the PCR amplicons were visualized and accurately sized on an Applied Biosystems Genetic Analyzer 3130xl (Applied Biosystems, Inc., Foster City, CA) (Fig. 1). PCR was performed in 25$\mu \mathrm{l}$ volumes containing approximately $50 \mathrm{ng}$ of template DNA, Taq 2X Master Mix (New England BioLabs, Ipswich, MA), and 0.2 $\mu \mathrm{M}$ forward (fluorescently labeled) and reverse primers (Integrated DNA Technologies, Coralville, IA). Cycling conditions consisted of 1 min initial denaturation at $95^{\circ} \mathrm{C}$, followed by 36 cycles of $30 \mathrm{~s}$ denaturation at $95^{\circ} \mathrm{C}, 30 \mathrm{~s}$ annealing at $65^{\circ} \mathrm{C}$, and $30 \mathrm{~s}$ extension at $72^{\circ} \mathrm{C}$. PCR was completed with a final extension for $10 \mathrm{~min}$ at $72^{\circ} \mathrm{C}$. Microsatellites were sized and scored on an Applied Biosystems Genetic Analyzer 3130xl equipped with a 36-cm capillary and POP-7 polymer. Before each run, the PCR product was diluted fivefold in sterile DI water, and $2 \mu$ of the diluted PCR product, $9.8 \mu \mathrm{l}$ of Hi-Di formamide (Applied Biosystems) and $0.2 \mu \mathrm{l}$ of GeneScan 500Liz size standard were added to each tube. Genemarker Software (version 1.7; SoftGenetics, State College, PA, USA) was used to analyze the data. Isolates with the same product sizes as FGVA4 for all three microsatellites were identified as the released clone. Microsatellites for FusSSR22, FusSSR23, and FusSSR27 are located on chromosomes 4, 2, and 1, respectively. Microsatellite locations on chromosomes were determined by performing BLAST queries against the genome of $F$. graminearum (5).

Seed assay to confirm clone. To assess whether FGVA4 contributed to infection in inoculated wheat subplots, a seed assay (SIC) was performed. Two wheat heads were collected randomly from 49 of the 50 inoculated subplots in 2011 (one of the infected plots had all the wheat removed in order to accommodate a spore release experiment, and consequently heads were not collected from this plot). In 2012, five wheat heads were collected randomly from all 50 of the inoculated subplots. Wheat kernels (seeds) were removed and cultured on FSM for 5 to 7 days, and a single isolate of $F$. graminearum from each head was purified and genotyped as described in the previous sections.

Statistical analyses. Data were analyzed for each year (2011 and 2012), and for both years combined (2011 and 2012). To quantify the relationship between recovery rate of the released clone and distance from the source, logistic regression was performed $(15,39)$. Odds were calculated for 28 sampling locations for 2011 and 40 sampling locations for 2012 where:

$$
\operatorname{odds}=\hat{p} /(1-\hat{p})
$$

$\hat{p}=\frac{\text { Total number of released clones that were captured }}{\text { Total number of Fusarium graminearum clones }}=$ recovery rate

The Logistic regression model is

$$
\text { Log odds }=\beta_{0}+\beta_{1} * x
$$

where $x$ is the distance from the source $(\mathrm{m}), \beta_{0}$ is the intercept coefficient, and $\beta_{1}$ is the slope coefficient.

$$
\begin{gathered}
\text { odds }=\frac{\hat{p}}{1-\hat{p}}=e^{\beta_{0}+\beta_{1}{ }^{*} x} \\
\hat{p}=\frac{e^{\beta_{0}+\beta_{1}{ }^{* x}}}{1+e^{\beta_{0}+\beta_{1}^{*} x^{*}}}
\end{gathered}
$$

All of the statistical analyses were performed using JMP System for Windows (Release 9, SAS Institute Inc., Cary, NC), R, and SAS (Release 9.2, SAS Institute).

\section{Results}

Field collections. In 2011, 12,409 viable Fusarium colonies were recovered over 14 consecutive days at distances up to $500 \mathrm{~m}$ from the center of the inoculum source; $77.4 \%(9,607 / 12,409)$ of the colonies were collected at night (1900 to 0700) and $22.6 \%$ $(2,802 / 12,409)$ were collected during the day (0700 to 1900). Of the 12,409 isolates recovered, 2,358 were subcultured on $1 / 4$ strength PDA for tentative identification of $F$. graminearum; 1,055 were tentatively identified as $F$. graminearum, and $790 \mathrm{~F}$. graminearum isolates were analyzed for the released clone using microsatellites.

In 2012, 6,283 viable Fusarium isolates were recovered over 19 consecutive days at distances up to $1,000 \mathrm{~m}$ from the center of the inoculum source; $63.3 \%(3,976 / 6,283)$ of the colonies were collected at night (1900 to 0700), and $36.7 \%(2,307 / 6,283)$ were collected during the day (0700 to 1100$)$. Of the 6,283 colonies recovered, 3,551 were subcultured on 1/4-strength PDA for tentative identification of $F$. graminearum. Three hundred and eighty-two colonies were tentatively identified as $F$. graminearum, and 237 of the $F$. graminearum isolates were analyzed to identify whether they were the released clone using the microsatellite markers.

Validation of microsatellite analysis. Prior to analyzing field collections for microsatellites, genomic DNA was obtained from $12 \mathrm{~F}$. graminearum samples from Vogelgsang et al. (46) to serve as positive controls for each of the selected microsatellites. All 12 control samples produced amplicon sizes identical to those produced by Vogelgsang et al. (46) (e.g., Fig. 1).

Sampling prior to clone release. Field samples were collected from 26 April to 2 May 2011 to assess background populations of
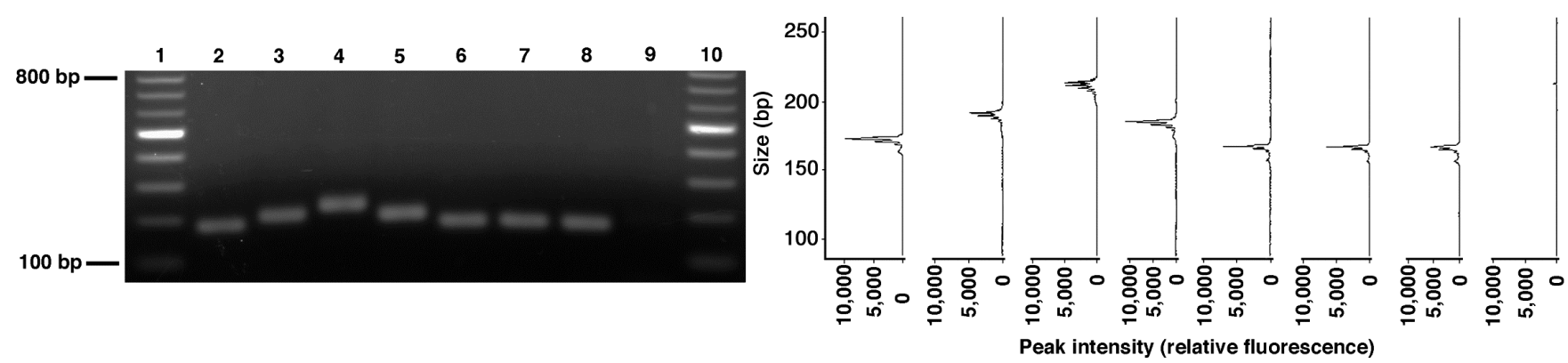

Fig. 1. Polymerase chain reaction (PCR) products (left) and electropherograms (right) for eight different isolates of Fusarium graminearum using the FusSSR23 primers. Lanes 1-10 represent the following in order from left to right: 100 bp mass ladder, isolates HUGR2, 11669, 4528, 6473 (46), the released clone FGVA4, an unknown isolate collected $500 \mathrm{~m}$ from the source, an unknown isolate collected $0 \mathrm{~m}$ (in the source) from the source, negative control (no template DNA), and $100 \mathrm{bp}$ mass ladder. Electropherograms (right) show product sizes of 173, 191, 213, 185, 167, 167, and $167 \mathrm{bp}$, which correspond to the same isolates listed for lanes 2 through 8 . The product sizes for isolates HUGR2, 11669, 4528, and 6473 are identical to those obtained by Vogelgsang et al. (46); S. Vogelgsang, personal communication), and the unknown isolates produced the same product size as the released isolate FGVA4. 
the released clone (FGVA4) prior to its release in 2011 (the field was inoculated on 2 May 2011). Fusarium colonies (1,985) were recovered during this interval, and 1,037 colonies were subcultured on $1 / 4$-strength PDA for tentative identification of $F$. graminearum. Of these, 283 were tentatively identified as $F$. graminearum and 141 isolates were analyzed to identify whether they were the released clone using the microsatellite markers. Three of these isolates $(2.1 \%, 3 / 141)$ produced amplicon sizes identical to the released clone at all three microsatellites and thus could not be distinguished from the released clone. Results from the microsatellite analysis for the 141 isolates analyzed for the background populations collected prior to the released clone yielded seven unique amplicons for FusSSR22, 26 unique amplicons for FusSSR23, and 27 unique amplicons for FusSSR27 (Tables 2 and 3). The allele frequencies for amplicon sizes for the released clone for FusSSR22 (200 bp), FusSSR23 (167 bp), and FusSSR27 (183 bp) were 0.614, 0.035 , and 0.156 , respectively (Table 2 ). Based on the allele frequencies calculated from the 141 background isolates collected prior to the release of the clone, $0.34 \%(0.614 * 0.035 * 0.156)$ of the isolates identified as the released clone would be expected to be false positives.

Field samples were collected from 9 to 12 April 2012 to assess background populations of the released clone (FGVA4) prior to its release in 2012 (the field was inoculated on 16 April 2012). Eighty-nine Fusarium colonies were recovered during this interval and subcultured on $1 / 4$-strength PDA for tentative identification of $F$. graminearum. Of these, seven were tentatively identified as $F$. graminearum and analyzed for microsatellite markers. None of these isolates $(0 \%, 0 / 7)$ produced amplicon sizes identical to the released clone at all three microsatellite loci.

Sampling after the release of the clone. Of the 790 isolates analyzed for the three microsatellites in $2011,22.1 \%(175 / 790)$ produced amplicons that were identical to the released clone for all three microsatellites and were considered to be the released clone. The remaining $77.9 \%(615 / 790)$ did not produce amplicons that were identical to the released clone for one or more microsatellites and were determined not to be the released clone, likely representing background populations from other sources $(19,20)$.

Of the 237 isolates analyzed for the three microsatellites in $2012,40.5 \%$ (96/237) produced amplicons that were identical to the released clone at all three microsatellites and were considered to be the released clone. The remaining 59.5\% (141/237) did not produce amplicons that were identical to the released clone at one or more microsatellites.

Comparisons between background populations collected prior to and during the release of the clone. To examine potential differences in allele frequencies between the background populations collected prior to and during release of the clone, 141 background isolates that were collected during the release of the clone were randomly selected for further analysis (Table 3). This was the same number of isolates analyzed from the background populations collected prior to release of the clone, and thus allowed us to compare identical sample sizes. An analysis of the population after release of FGVA4 of the polymorphisms for each microsatel-

Table 2. Allele frequency of three microsatellites in background populations of Fusarium graminearum prior to the release (26 April to 2 May 2011$)$ and during the release (12 May to 25 May 2011) of clone FGVA4 in the 2011 field experiment

\begin{tabular}{|c|c|c|c|c|c|}
\hline $\begin{array}{l}\text { Prior to or during } \\
\text { release of the clone }\end{array}$ & Microsatellite & $\begin{array}{l}\text { Amplicon size of } \\
\text { released clone (bp) }\end{array}$ & Number of amplicons & $\begin{array}{l}\text { Allele frequency of } \\
\text { the released clone }\end{array}$ & Sample size $^{\mathrm{a}}$ \\
\hline Prior & FusSSR22 & 200 & 7 & 0.614 & $140^{\mathrm{b}}$ \\
\hline During & FusSSR22 & 200 & 11 & 0.525 & 141 \\
\hline Prior & FusSSR 23 & 167 & 26 & 0.035 & 141 \\
\hline During & FusSSR23 & 167 & 32 & 0.071 & 141 \\
\hline Prior & FusSSR27 & 183 & 27 & 0.156 & $135^{\mathrm{c}}$ \\
\hline During & FusSSR27 & 183 & 30 & 0.227 & 141 \\
\hline
\end{tabular}

a One hundred forty-one isolates of $F$. graminearum were analyzed for background populations collected prior to release of the clone, which represented all (141/141) of the background isolates analyzed for this interval. One hundred forty-one isolates (the same number from background populations prior to release) were selected at random from background populations collected during the release of the clone, which represented 23\% (141/617) of the background isolates analyzed during this interval.

${ }^{\mathrm{b}}$ No amplicon was obtained for 1 isolate after three independent polymerase chain reactions.

${ }^{\mathrm{c}}$ No amplicon was obtained for 6 isolates after three independent polymerase chain reactions.

Table 3. Background isolates analyzed for three microsatellites (FuSSSR22, FusSSR23, and FusSSR27) from collections prior to the release (26 April to 2 May 2011) and during the release (12 May to 25 May 2011) of clone FGVA4 for the 2011 field experiment

\begin{tabular}{|c|c|c|c|c|c|c|}
\hline \multirow{2}{*}{$\begin{array}{l}\text { Prior to or during } \\
\text { release of the clone } \mathrm{e}^{\mathrm{a}}\end{array}$} & \multirow[b]{2}{*}{ Sampling date } & \multicolumn{4}{|c|}{ Number of isolates analyzed at sampling distance (m) } & \multirow{2}{*}{$\begin{array}{l}\text { Total background Fusarium } \\
\text { graminearum isolates analyzed }\end{array}$} \\
\hline & & o (in the source) & 100 & 250 & 500 & \\
\hline Prior & 26 April 2011 & 1 & 13 & 10 & 6 & 30 \\
\hline Prior & 27 April 2011 & 3 & 17 & 11 & 4 & 35 \\
\hline Prior & 29 April 2011 & 3 & 19 & 15 & 11 & 48 \\
\hline Prior & 2 May 2011 & 2 & 12 & 7 & 7 & 28 \\
\hline During & 12 May 2011 & 1 & 8 & 2 & 3 & 14 \\
\hline During & 13 May 2011 & 0 & 4 & 5 & 3 & 12 \\
\hline During & 14 May 2011 & 1 & 7 & 2 & 3 & 13 \\
\hline During & 15 Мау 2011 & 4 & 1 & 5 & 6 & 16 \\
\hline During & 16 May 2011 & 0 & 6 & 2 & 7 & 15 \\
\hline During & 17 Мау 2011 & 0 & 8 & 5 & 5 & 18 \\
\hline During & 18 May 2011 & 3 & 3 & 4 & 2 & 12 \\
\hline During & 19 Мау 2011 & 0 & 0 & 0 & 0 & 0 \\
\hline During & 20 Мау 2011 & 0 & 5 & 10 & 2 & 17 \\
\hline During & 21 May 2011 & 0 & 4 & 3 & 1 & 8 \\
\hline During & 22 Мау 2011 & 1 & 3 & 5 & 5 & 14 \\
\hline During & 23 May 2011 & 0 & 2 & 0 & 0 & 2 \\
\hline During & 24 May 2011 & 0 & 0 & 0 & 0 & 0 \\
\hline During & 25 May 2011 & 0 & 0 & 0 & 0 & 0 \\
\hline
\end{tabular}

${ }^{a}$ One hundred forty-one isolates from each of these populations were selected for comparisons of background populations; isolates for the first set represented all of the isolates analyzed prior to the release of the clone, and isolates for the second set were chosen at random from the larger population of isolates across multiple sampling days and distances from the source. 
lite showed that 11 amplicon sizes were observed for FusSSR22, 32 for FusSSR23, and 30 for FusSSR27 (Tables 2 and 3). The allele frequencies for amplicon sizes for the released clone FusSSR22 (200 bp), FusSSR23 (167 bp), and FusSSR27 (183 bp) were $0.525,0.071$, and 0.227 , respectively (Table 2 ). Based on the allele frequencies calculated from the 141 background isolates collected during release of the clone, $0.85 \%(0.525 * 0.071 *$ 0.227 ) of the isolates identified as the released clone would be expected to be false positives.

Day and night collection of $\boldsymbol{F}$. graminearum. In 2011, most of the $F$. graminearum isolates analyzed for microsatellites (clonal
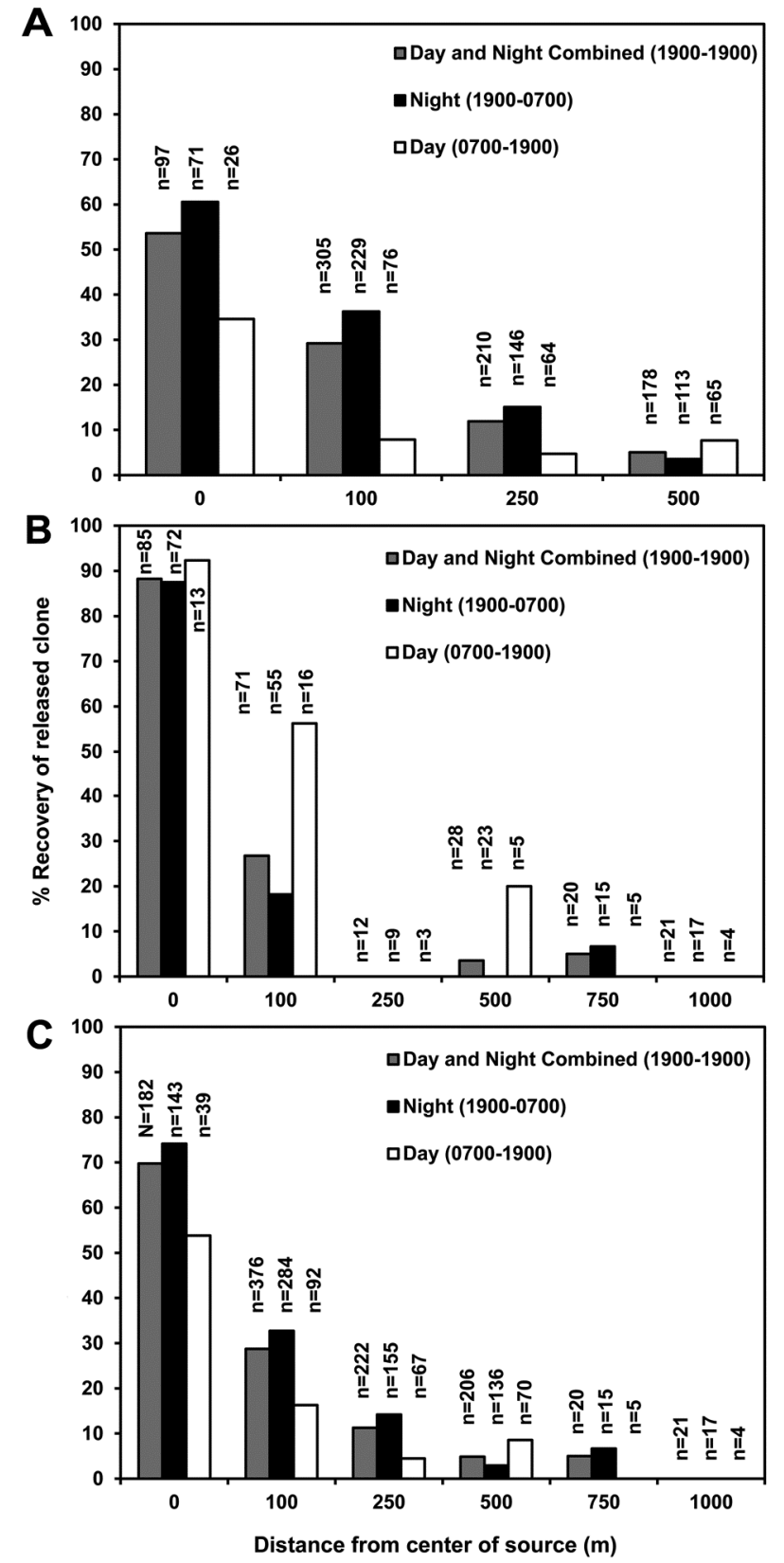

Fig. 2. Recovery of released clone FGVA4 during the night, day, and night and day combined at different distances from the center of a $3,716 \mathrm{~m}^{2}$ clonal source of inoculum for 2011 (A), 2012 (B), and 2011 and 2012 data combined (C). Samples were collected during the day (0700-1900) and night (1900-0700) 12-25 May in the 2011 season. Samples were collected during the day (0700-1100) and night (19000700) 26 April-14 May in the 2012 season. Sample sizes (n) listed above each of the bars in the figure represent total isolates from which the proportion was calculated. The majority of FGVA4 was recaptured during the night (1900-0700), and recovery of FGVA4 decreased at greater distances from the source. The distance of $0 \mathrm{~m}$ from the center of the source refers to the three samplers placed in the inoculum source. populations) were collected during the night (70.8\% [559/790], 1900 to 0700$)$ and early morning (24.5\% [194/790], 0700 to 1100$)$ hours (Fig. 2). The remainder of the isolates analyzed for microsatellites were collected during the day, 3\% (21/792) for 1100 to 1500 and $2 \%(16 / 790)$ for 1500 to 1900 .

Based on data collected in 2011, samples were only collected during the night (1900 to 0700$)$ and early morning (0700 to 1100$)$ hours during the 2012 field season. In 2012, 81\% (191/237) of the $F$. graminearum isolates analyzed for microsatellites (clonal populations) were collected during the night (1900 to 0700) and 19\% (46/237) were collected during the early morning (0700 to 1100$)$.

Long-distance transport of released clone. The majority of the released clone was recaptured during the night (1900 to 0700) and early morning (0700 to 1100 ) sampling periods, with a decrease at increasing distances from the source for 2011 and 2012 (Fig. 2). When combining data in both years, $74.1 \%$ (106/143), $32.7 \%$ (93/284), 14.2\% (22/155), 2.9\% (4/136), 6.7\% (1/15), and $0 \%$ $(0 / 17)$ recovery of the released clone occurred at 0 (in the source), $100,250,500,750$, and $1,000 \mathrm{~m}$, respectively, from the source during the night (1900 to 0700). There was a 53.8\% (21/39), $16.3 \%$ (15/92), $4.5 \%$ (3/67), $8.6 \%(6 / 70), 0 \%(0 / 5)$, and $0 \%(0 / 4)$ recovery of the released clone at 0 (in the source), 100, 250, 500, 750, and $1,000 \mathrm{~m}$, respectively, from the source during the day (0700 to 1100) (Fig. 2).

Recovery of the released clone followed a logistic regression model (Table 4; Fig. 3) $(15,39)$, and was significant $(P<0.041$ for all slope term scenarios) as a function of distance from the source of inoculum. The slope term $\left(\beta_{1}\right)$ in the regression analysis was always negative (Table 4), indicating a decrease in the recovery of the released clone as the distance from the source of inoculum increased. The total number of clones was fixed at four distances $(0,100,250$, and $500 \mathrm{~m})$ for 2011 and two additional distances (750 and $1,000 \mathrm{~m}$ ) for 2012 .

Seed assay. In 2011, two wheat heads from 49 subplots were analyzed for presence of the released clone. There was an $84.7 \%$ (83/98) recovery of the released clone in the seed assay. Each of the plots analyzed had at least one wheat head infected by the released clone. In 2012, of the 250 heads subjected to seed assay for the released clone there was an $80 \%$ (141/177) recovery of the released clone.

\section{Discussion}

Research is needed to understand the long-distance movement ( $>100 \mathrm{~m}$ ) of $F$. graminearum from field-scale sources of inoculum. Such information may be useful for predicting epidemics of FHB and targeting the early application of fungicides. In the present study, the movement of a unique strain of $F$. graminearum from a 0.372 ha inoculum source was monitored over two field seasons. The source was designed to represent a commercial cropping scenario. Microsatellite markers were used to unambiguously track the movement of the released clone at distances up to $1,000 \mathrm{~m}$ from the source, and we recovered the released clone up to $750 \mathrm{~m}$ from the inoculum source. Our work extends the research of Fernando et al. (14) and Keller et al. $(19,20)$ by monitoring the transport of $F$. graminearum over a greater distance from a known source of inoculum and using microsatellite markers to unambiguously track the movement of the fungus.

Recovery of the released clone of $F$. graminearum decreased at greater distances from the source. Keller et al. $(19,20)$ observed a similar trend from small area sources of inoculum, but with a much steeper gradient of recovery over much shorter distances. The furthest distance sampled from the released source was $30 \mathrm{~m}$, where the recovery was $\leq 1 \%$ for all scenarios. It is important to note that Keller et al. $(19,20)$ released small amounts of inoculum $(<500 \mathrm{~g})$ in $0.84 \mathrm{~m}$ diameter circular plots that could have contributed to a steep drop-off of the released clone recovered, compared to our experiment where there was a 0.372 ha source (estimated 78,000 g of corn stalks). Our aim was to collect viable spores deposited out of the atmosphere onto plates of selective medium, whereas Keller et al. $(19,20)$ attempted to collect the released clone from diseased 
wheat heads. Thus, subtle differences between the results of these studies might be attributed to factors affecting the infection and recovery of the released clone in the different experimental systems. One of the major limitations with our experimental design was lack of knowledge of the inoculum source variability, as different microclimates across the field could drive different rates of spore release. Additionally, a large proportion of spores released are not expected to escape the turbulent boundary layer and thus will not be able to travel over long distances (32).

The released clone of $F$. graminearum was recovered up to 750 $\mathrm{m}$ from the field scale source; to our knowledge this is the first report demonstrating the transport of $F$. graminearum spores at least $750 \mathrm{~m}$ from a known field-scale source. Although advances have been made in mathematically modeling the long-distance transport of plant pathogens, some of these models have not been validated experimentally. The approach used here could help validate transport models. Assuming a Gaussian plume of spores released from the source (1-4), the dispersal kernel should drop off quickly at greater downwind distances from the source (as a power law times an exponential). Sampling at even greater distances, in the tail of the dispersal kernel, poses a challenge since there is expected to be a dilution in the concentration of spores at downwind distances from the source and the sampling area effectively becomes much larger. This could be one possible explanation for not capturing the released clone at $1,000 \mathrm{~m}$ (spores of the released clone may have traveled $1,000 \mathrm{~m}$ or more, but we did not recover them at our sampling sites). Experimentally determining the "fat tail" of the dispersal kernel is important since slight changes in the long-distance dispersal tail can lead to large changes in predicted spread rates, since rare long-distance dispersal events described by the tails are the dominant factor in determining the rate of disease spread $(17,22)$.

The release and recapture studies were done in replicates over two field seasons during 2011 and 2012. In 2011, we attempted to recapture the released clone up to $500 \mathrm{~m}$ from the field scale source of inoculum. Since we were able to recover the clone at 500 $\mathrm{m}$ in 2011 , the sampling distances $(750$ and $1,000 \mathrm{~m})$ and the number of samplers (40) was increased during the second year of experimentation. In both years, we observed a similar trend of a decrease in recapture of the release clone with greater distance from the source of inoculum. Recovery of the released clone followed a logistic regression model, and was significant $(P<0.041$ for all slope term scenarios) as a function of distance from the source of inoculum. Logistic regression models are commonly used in epidemiology to model dispersal of pathogens and disease progression $(6,15,26)$. The slope term $\left(\beta_{1}\right)$ in our regression analysis was al- ways negative, indicating a decrease in the recovery of the released clone as the distance from the source of inoculum increased. Additionally, both in 2011 and 2012 the majority of the released clone was recovered during the night (1900 to 0700). A major difference observed between 2011 and 2012 was the steep drop-off of the dispersal kernel in 2012 compared to 2011. In 2012, there was a much greater recovery of the released clone (88\%) compared to $2011(53 \%)$ at $0 \mathrm{~m}$ (in the source). However, when the samplers were located $100 \mathrm{~m}$ from the source of inoculum, recovery of the released clone in 2012 (26\%) was less than the recovery in 2011 (29\%). Differences in meteorological conditions between years may have influenced some of these trends $(1,2)$, in particular environmental factors that govern spore production and release for the fungus (13,28,43-45). Aylor et al. (2,3) discusses the importance of rainfall in "washing out" spores from a column of air. One possible explanation for the differences observed between 2011 and 2012 is that ascospore release and rainfall need to be coupled events to have a high recovery of the released clone at sampling distances outside the field scale source of inoculum, yielding a fat tail in the dispersal kernel as seen in 2011. This idea is the basis of a recently

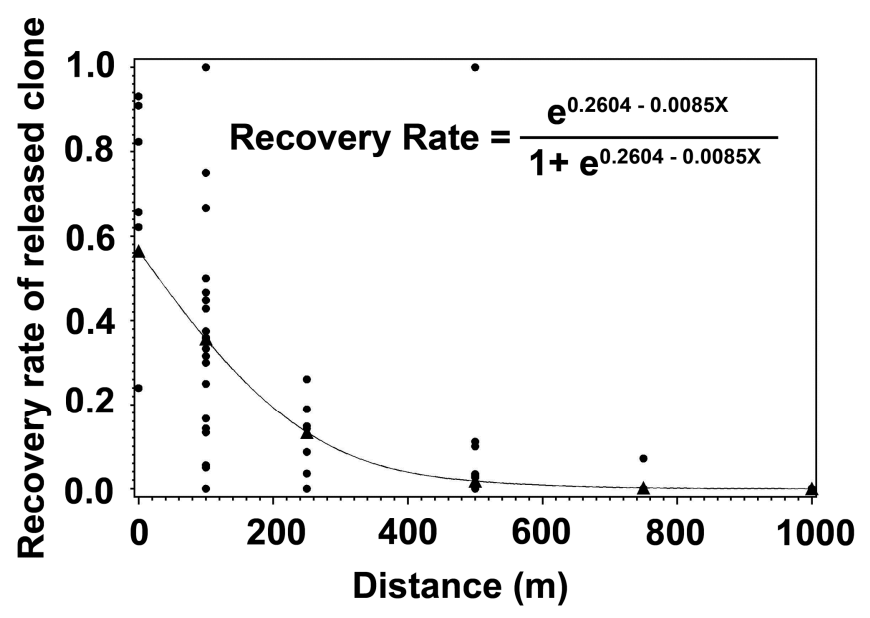

Fig. 3. Logistic regression plot of day and night data for 2011 and 2012 combined. Accompanying model statistics are reported in the text of the manuscript for each sampling scenario (2011 day, 2011 night, 2011 day and night combined, 2012 day, 2012 night, 2012 day and night combined, 2011/2012 day, 2011/2012 night, 2011/2012 day and night combined); however, a similar regression trend was observed for each case. The distance of $0 \mathrm{~m}$ from the center of the source refers to the three samplers placed in the source of inoculum.

Table 4. Model parameters for logistic regression analysis of recovery of released clone FGVA4 of Fusarium graminearum in 2011 (12 May-25 May) and 2012 (26 April-14 May) $^{\mathrm{a}}$

\begin{tabular}{|c|c|c|c|c|c|c|c|}
\hline \multirow{2}{*}{$\frac{\text { Sampling period }}{\text { Day + Night }}$} & \multirow{2}{*}{$\begin{array}{l}\text { Year } \\
2011\end{array}$} & \multirow{2}{*}{$\frac{\text { Parameter }}{\beta_{0}}$} & \multirow{2}{*}{$\frac{\text { Estimate }}{-0.1445}$} & \multicolumn{2}{|c|}{ Wald $95 \%$ confidence limits } & \multirow{2}{*}{$\begin{array}{c}\text { Wald Chi-square } \\
1.1000\end{array}$} & \multirow{2}{*}{$\frac{\boldsymbol{P}>\mathbf{C h i S q}^{\mathbf{b}}}{0.2950}$} \\
\hline & & & & -0.4149 & 0.1259 & & \\
\hline & & $\beta_{1}$ & -0.0066 & -0.0082 & -0.0051 & 68.3000 & $<0.0001$ \\
\hline & 2012 & $\beta_{0}$ & 1.2300 & 0.7483 & 1.7117 & 25.0500 & $<0.0001$ \\
\hline & & $\beta_{1}$ & -0.0157 & -0.0216 & -0.0099 & 28.2100 & $<0.0001$ \\
\hline & 2011 and 2012 & $\beta_{0}$ & 0.2604 & 0.0365 & 0.4843 & 5.2000 & 0.0226 \\
\hline & & $\beta_{1}$ & -0.0085 & -0.0100 & -0.0070 & 118.0300 & $<0.0001$ \\
\hline \multirow[t]{6}{*}{ Night } & 2011 & $\beta_{0}$ & 0.2672 & -0.0494 & 0.5838 & 2.7400 & 0.0981 \\
\hline & & $\beta_{1}$ & -0.0078 & -0.0097 & -0.0058 & 61.2000 & $<0.0001$ \\
\hline & 2012 & $\beta_{0}$ & 1.3149 & 0.7579 & 1.8718 & 21.4100 & $<0.0001$ \\
\hline & & $\beta_{1}$ & -0.0211 & -0.0287 & -0.0135 & 29.6800 & $<0.0001$ \\
\hline & 2011 and 2012 & $\beta_{0}$ & 0.5342 & 0.2717 & 0.7968 & 15.9100 & $<0.0001$ \\
\hline & & $\beta_{1}$ & -0.0095 & -0.0114 & -0.0076 & 96.3200 & $<0.0001$ \\
\hline \multirow[t]{6}{*}{ Day } & 2011 & $\beta_{0}$ & -1.6026 & -2.2595 & -0.9458 & 22.8700 & $<0.0001$ \\
\hline & & $\beta_{1}$ & -0.0030 & -0.0058 & -0.0001 & 4.1700 & 0.0411 \\
\hline & 2012 & $\beta_{0}$ & 1.4653 & 0.4372 & 2.4934 & 7.8000 & 0.0052 \\
\hline & & $\beta_{1}$ & -0.0089 & -0.0157 & -0.0021 & 6.6600 & 0.0099 \\
\hline & 2011 and 2012 & $\beta_{0}$ & -0.6540 & -1.1274 & -0.1806 & 7.3300 & 0.0068 \\
\hline & & $\beta_{1}$ & -0.0055 & -0.0081 & -0.0029 & 17.6700 & $<0.0001$ \\
\hline
\end{tabular}

\footnotetext{
a Analysis included data collected during the day (0700-1900), night (1900-0700), and day and night combined (0700-0700).
}

b The fitted model was: Log odds $=\beta_{0}+\beta_{1} *$ Distance from the source. 
submitted manuscript (30) to validate spore transport models (1-4) with the experimental results reported here.

The majority (87\% for $2011 ; 77 \%$ for 2012) of the released clone was collected during the night (1900 to 0700). These results are consistent with previous literature examining the release dynamics of spores from artificially inoculated plots. Paulitz (28) and Fernando et al. (13) found that the greatest aerial concentration of spores at a spore sampler located $1.5 \mathrm{~m}$ from an inoculated plot occurred between 2000 and 0800 , with a low number of spores present between 1200 and 1600, suggesting a night-time release of ascospores from perithecia. Schmale et al. (35) studied the deposition of $F$. graminearum from unknown sources and found the majority of spores were deposited at night with peak deposition between 0400 and 0600. Previous research also showed that the majority (91\%) of $F$. graminearum was deposited in corn canopies during the night (34). Our results are consistent with this finding; the majority (95\%) of F. graminearum were collected from 1900 to 1100. It is possible that deposition rates are higher at night, due to a rapid cooling of earth's surface compared to the atmosphere and an inversion layer being formed due to a downward transfer of heat from the atmosphere to the surface (27). Differences in meteorological conditions during the day and night could help explain small variations in the recovery of the released clone during the day and night. There has been speculation that there is an uncoupling between the release and deposition of $F$. graminearum spores, with conditions for spore release being most favorable during the day and deposition occurring at night $(23,32,35)$.

The majority ( $85 \%$ for $2011 ; 80 \%$ for 2012 ) of wheat heads analyzed in the seed assay were infected with the released clone, confirming the ability of this clone to cause FHB in wheat. In 2011, there was, however, a higher recovery rate of the released clone in the seed assay compared to collections on sample plates in the source $(0 \mathrm{~m})$. One possible explanation for this difference is that the seed assay reflects cumulative exposure of wheat heads to spores over a long interval of time (weeks), whereas the sampling plates were only exposed for a short interval of time (hours). We did not observe this trend in 2012, the recovery rate of the released clone for the sampling plates in the source $(0 \mathrm{~m})$ was slightly higher $(88 \%)$ than for the seed assay. It is possible that all of the wheat heads were infected with the released clone, but we only sampled and analyzed a single isolate from each infected head. Zeller et al. (47) found that it is possible for multiple isolates of $F$. graminearum to infect a single wheat head and cause FHB.

Previous work has shown that $F$. graminearum survives as a saprophyte in crop debris (wheat, corn, and barley, among others) from the previous growing season (12). Dill-Macky and Jones (10) found that FHB incidence and severity were highest when wheat followed a susceptible host (corn) than when wheat followed a nonsusceptible host (soybeans). Additionally, Dill-Macky and Jones (10) suggested that a reduced-till system contributed to increased DON levels due to $F$. graminearum being able to survive as a saprophyte on crop debris remaining from the previous growing season and acting as a local source of inoculum. This work was further validated by Keller et al. $(19,20)$, who observed that local sources of inoculum within a wheat field contributed to FHB. The results obtained in this study highlight the potential contributions of both local (within field) and more distant sources (e.g., those that are transported hundreds of meters or further from neighboring fields and/or regions) to FHB. The high recovery of the released clone in the seed assay further validates the role of within-field inoculum sources in causing FHB. However, recovery of the released clone at distant sources from the inoculated field $(>100 \mathrm{~m})$ suggests that distant sources of $F$. graminearum have the potential to contribute to FHB.

Comparisons between the 141 background isolates analyzed from collections prior to the release of the clone and the 141 isolates analyzed from collections during the release of the clone demonstrated that background populations were similar, at least in terms of the number of unique amplicons and allele frequencies for the three microsatellites. The number of unique amplicon sizes was comparable for both populations for all three microsatellites ( 7 and 11 unique amplicons for FusSSR22 prior to and during release, respectively; 26 and 32 unique amplicons for FusSSR23 prior to and during release, respectively; and 27 and 30 unique amplicons for FusSSR27 prior to and during release, respectively), and the allele frequencies were similar (0.614 and 0.525 for FusSSR22 prior to and during release, respectively; 0.035 and 0.071 for FusSSR23 prior to and during release, respectively; and 0.156 and 0.227 for FusSSR27 prior to and during release, respectively). These results support the hypothesis that background populations of $F$. graminearum collected from the atmosphere are well-mixed $(36,37)$. Moreover, this analysis demonstrates that the possibility of having a false positive was less than $1 \%$ for both populations $(0.34 \%$ for collections prior to the release of the clone and $0.85 \%$ during the release of the clone). Thus, isolates producing amplicons identical to the released clone at all three microsatellites would be identified as the released clone.

Data from background sampling prior to the release of the clone in the field during 2011 showed that the released clone appeared to be present in about 2\% (3/141) of the isolates. In 2012, only one $F$. graminearum isolate was captured during background sampling, and this isolate was not the released clone. Thus, the potential contribution of background sources of the clone (i.e., sources other than the source we released) is possible, but very small. Vogelgsang et al. (46) proposed 15 different microsatellite markers for $F$. graminearum. In an attempt to resolve the three background isolates whose allelic sizes were identical to the released clone for the three microsatellites studied, we examined all 15 microsatellite markers proposed by Vogelgsang et al. (46) and compared their allelic sizes to the released clone (data not shown). Two of the background isolates showed identical allelic sizes to the released clone at all 15 microsatellite markers, but one of the background isolates yielded a different product, suggesting that this third isolate was not the released clone. Thus, the inclusion of additional microsatellite markers (beyond the three included in this study) has the potential to further decrease the markedly low percentage of false positives.

Future work may include the use of a meteorological-based mathematical model to predict and validate the local (spores from a released source) and regional (spores from other sources) transport of $F$. graminearum. Such work may help improve management practices for FHB and contribute to the development of early warning systems for the spread of $F$. graminearum and other important plant pathogens. For example, a current online prediction tool for FHB is available (9). The main considerations of the current FHB risk assessment tool $(7,8)$ are environmental factors such as temperature, precipitation, and relative humidity to determine the risk of an FHB outbreak. In addition to environmental factors, the risk assessment tool also considers disease reports from local extension agents. Although environmental factors are the main components of disease development of FHB on wheat and barley, the current risk assessment tool does not include the ability to predict the movement of $F$. graminearum spores from potential source areas. Previous literature has shown that $F$. graminearum spores are ubiquitous in the planetary boundary layer of the atmosphere (24), and may be transported across broad geographical regions (3). Future work may include the development of mathematical models to predict the long-distance transport of $F$. graminearum, and these models could leverage release-recapture experiments such as those described here to validate these models. Such models could provide a powerful prediction tool and allow producers of small grains to employ improved disease management practices for FHB, such as the early application of appropriate fungicides. Moreover, this work could find immediate application in the development of model-based early warning systems for the spread of other highrisk plant pathogens such as Peronospora tabacina, causal agent of tobacco blue mold, (4) Phakopsora pachyrhizi, causal agent of Asian soybean rust $(4,21)$, and Puccinia graminis f. sp. tritici, causal agent of wheat stem rust (40). 


\section{Acknowledgments}

This material is based upon work supported by the National Science Foundation under Grant Numbers DEB-0919088 (Atmospheric transport barriers and the biological invasion of toxigenic fungi in the genus Fusarium), CMMI1100263 (Dynamical mechanisms influencing the population structure of airborne pathogens: Theory and observations), DGE-0966125 (IGERT: MultiScale Transport in Environmental and Physiological Systems (MultiSTEPS)), and Virginia Small Grains Board proposal numbers 11-2660-06 and 12-2562-05 (Tracking the long-distance transport of the fungus that causes Fusarium head blight in wheat and barley). Any opinions, findings, and conclusions or recommendations expressed in this material are those of the authors and do not necessarily reflect the views of the National Science Foundation or the Virginia Small Grains Board. We are grateful to $\mathrm{S}$. Vogelgsang for providing DNA from $F$. graminearum to serve as positive controls for the microsatellite experiments. We would like to thank T. Welling, Z. Bair, N. Szanyi, and Z. Upchurch for excellent technical assistance in the laboratory and field. We would also like to acknowledge LISA (Laboratory for Interdisciplinary Statistical Analysis) of Virginia Polytechnic Institute and State University for statistical support.

\section{Literature Cited}

1. Aylor, D. E. 1986. A framework for examining inter-regional aerial transport of fungal spores. Agric. For. Meteorol. 38:263-288.

2. Aylor, D. E. 1999. Biophysical scaling and the passive dispersal of fungus spores: Relationship to integrated pest management strategies. Agric. For. Meteorol. 97:275-292.

3. Aylor, D. E., and Sutton, T. B. 1992. Release of Venturia inaequalis ascospores during unsteady rain: Relationship to spore transport and deposition. Phytopathology 82:532-540.

4. Aylor, D. E., Taylor, G. S., and Raynor, G. S. 1982. Long-range transport of tobacco blue mold spores. Agric. Meteorol. 27:217-232.

5. Cuomo, C. A., Güldener, U., Xu, J.-R., Trail, F., Turgeon, B. G., Di Pietro, A., Walton, J. D., Ma, L.-J., Baker, S. E., and Rep, M. 2007. The Fusarium graminearum genome reveals a link between localized polymorphism and pathogen specialization. Science 317:1400-1402.

6. Cushman, J., and Meentemeyer, R. K. 2008. Multi-scale patterns of human activity and the incidence of an exotic forest pathogen. J. Ecol. 96:766-776.

7. De Wolf, E. D., Madden, L. V., and Lipps, P. E. 2003. Risk assessment models for wheat Fusarium head blight epidemics based on within-season weather data. Phytopathology 93:428-435.

8. Del Ponte, E. M., Fernandes, J. M. C., Pavan, W., and Baethgen, W. E. 2009. A model-based assessment of the impacts of climate variability on Fusarium head blight seasonal risk in southern Brazil. J. Phytopathol. 157:675-681

9. DeWolf, E., Molineros, J., Paul, P., Madden, L., Shaner, G., Ali, S., Knight, P., Miller, S., Wisniewski, T., Miller, D., Crawford, D., Auer, T., Anderson, M., Ketchum, B., Brown, J., Akyuz, A., Carcoana, R., and Guinana, P. 2013. Fusarium head blight prediction center. Penn State, The Ohio State University, Kansas State University, and United States Wheat and Barley Scab Initiative: http://www.wheatscab.psu.edu/

10. Dill-Macky, R., and Jones, R. 2000. The effect of previous crop residues and tillage on Fusarium head blight of wheat. Plant Dis. 84:71-76.

11. Dufault, N., De Wolf, E., Lipps, P., and Madden, L. 2006. Role of temperature and moisture in the production and maturation of Gibberella zeae perithecia. Plant Dis. 90:637-644.

12. Fernandez, M. R. 1991. Recovery of Cochliobolus sativus and Fusarium graminearum from living and dead wheat and nongramineous winter crops in southern Brazil. Can. J. Bot. 69:1900-1906.

13. Fernando, W. G., Miller, J. D., Seaman, W. L., Seifert, K., and Paulitz, T. C. 2000. Daily and seasonal dynamics of airborne spores of Fusarium graminearum and other Fusarium species sampled over wheat plots. Can. J. Bot. 78:497-505.

14. Fernando, W. G. D., Paulitz, T. C., Seaman, W. L., Dutilleul, P., and Miller, J. D. 1997. Head blight gradients caused by from area sources of inoculum in wheat field plots. Phytopathology 87:414-421.

15. Hastings, A., Cuddington, K., Davies, K. F., Dugaw, C. J., Elmendorf, S., Freestone, A., Harrison, S., Holland, M., Lambrinos, J., and Malvadkar, U. 2005. The spatial spread of invasions: New developments in theory and evidence. Ecol. Lett. 8:91-101.

16. Herbek, J., and Lee, C. 2009. A comprehensive guide to wheat management in Kentucky. University of Kentucky Grain Crops Extension, Lexington, KY.

17. Higgins, S., Nathan, R., and Cain, M. 2003. Are long-distance dispersal events in plants usually caused by nonstandard means of dispersal? Ecology 84:1945-1956.

18. Isard, S. A., Gage, S. H., Comtois, P., and Russo, J. M. 2005. Principles of the atmospheric pathway for invasive species applied to soybean rust. Bioscience 55:851-861.

19. Keller, M. D., Thomason, W. E., and Schmale, D. G., III. 2011. The spread of a released clone of Gibberella zeae from different amounts of infested corn residue. Plant Dis. 95:1458-1464.

20. Keller, M. D., Waxman, K. D., Bergstrom, G. C., and Schmale, D. G., III.
2010. Local distance of wheat spike infection by released clones of Gibberella zeae disseminated from infested corn residue. Plant Dis. 94:11511155.

21. Krupa, S., Bowersox, V., Claybrooke, R., Barnes, C. W., Szabo, L., Harlin, K., and Kurle, J. 2006. Introduction of Asian soybean rust urediniospores into the midwestern United States-A case study. Plant Dis. 90:1254-1259.

22. Lewis, M. A. 1997. Variability, patchiness, and jump dispersal in the spread of an invading population. Pages 46-69 in: Spatial Ecology: The Role of Space in Population Dynamics and Interspecific Interactions. D. Tilman and P. Kareiva, eds. Princeton University Press, Princeton.

23. Maldonado-Ramirez, S. L. 2001. Aerobiology of the wheat scab fungus, Gibberella zeae: Discharge, atmospheric dispersal, and deposition of ascospores. Ph.D. thesis. Cornell University, Ithaca, NY.

24. Maldonado-Ramirez, S. L., Schmale, D. G., III, Shields, E. J., and Bergstrom, G. C. 2005. The relative abundance of viable spores of Gibberella zeae in the planetary boundary layer suggests the role of long-distance transport in regional epidemics of Fusarium head blight. Agric. For. Meteorol. 132:20-27.

25. McMullen, M., Jones, R., and Gallenberg, D. 1997. Scab of wheat and barley: A re-emerging disease of devastating impact. Plant Dis. 81:13401348 .

26. Mundt, C. C., Sackett, K. E., Wallace, L. D., Cowger, C., and Dudley, J. P. 2009. Aerial dispersal and multiple-scale spread of epidemic disease. EcoHealth 6:546-552.

27. Oke, T. R. 1987. Boundary layer climates. 2nd ed. Cambridge University Press, Cambridge, UK.

28. Paulitz, T. C. 1996. Diurnal release of ascospores by Gibberella zeae in inoculated wheat plots. Plant Dis. 80:674-678.

29. Paulitz, T. C. 1999. Fusarium head blight: A re-emerging disease. Phytoprotection (Québec) 80

30. Prussin, A. J., II. 2013. Monitoring and predicting the long distance transport of Fusarium graminearum, causal agent of Fusarium head blight in wheat and barley. Ph.D. thesis. Virginia Polytechnic Institute and State University, Blacksburg, VA.

31. Prussin, A. J., II, Ross, S. D., and Schmale, D. G., III. 2011. Tracking the long distance movement of Fusarium graminearum, causal agent of Fusarium head blight in wheat and barley. American Society of MicrobiologyGeneral Meeting, San Francisco, CA.

32. Schmale, D. G., III, Arntsen, Q. A., and Bergstrom, G. C. 2005. The forcible discharge distance of ascospores of Gibberella zeae. Can. J. Plant Pathol. 27:376-382.

33. Schmale, D. G., III, and Bergstrom, G. C. 2003. Fusarium head blight in wheat. The Plant Health Instructor. DOI:10.1094/PHI-I-2003-0612-01

34. Schmale, D. G., III, and Bergstrom, G. C. 2004. Spore deposition of the ear rot pathogen, Gibberella zeae, inside corn canopies. Can. J. Plant Pathol. 26:591-595.

35. Schmale, D. G., III, Bergstrom, G. C., and Shields, E. J. 2006. Night-time spore deposition of the fusarium head blight pathogen, Gibberella zeae, in rotational wheat fields. Can. J. Plant Pathol. 28:100-108.

36. Schmale, D. G., III, Leslie, J. F., Zeller, K. A., Saleh, A. A., Shields, E. J., and Bergstrom, G. C. 2006. Genetic structure of atmospheric populations of Gibberella zeae. Phytopathology 96:1021-1026.

37. Schmale, D. G., III, Ross, S., Fetters, T., Tallapragada, P., Wood-Jones, A. and Dingus, B. 2012. Isolates of Fusarium graminearum collected 40-320 meters above ground level cause Fusarium head blight in wheat and produce trichothecene mycotoxins. Aerobiologia 28:1-11.

38. Snijders, C. H. A. 1990. Fusarium head blight and mycotoxin contamination of wheat, a review. Eur. J. Plant Pathol. 96:187-198.

39. Stokes, M. E., Davis, M. E. S. C. S., Koch, G. G., and Davis, C. S. 2003 Categorical data analysis using the SAS system. SAS Institute, Cary, NC.

40. Stokstad, E. 2007. Deadly wheat fungus threatens world.s breadbaskets. Science 315:1786-1787.

41. Sutton, J. 1982. Epidemiology of wheat head blight and maize ear rot caused by Fusarium graminearum. Can. J. Plant Pathol. 4:195-209.

42. Trail, F., and Common, R. 2000. Perithecial development by Gibberella zeae: A light microscopy study. Mycologia 92(1):130-138.

43. Trail, F., Gaffoor, I., and Vogel, S. 2005. Ejection mechanics and trajectory of the ascospores of Gibberella zeae (anamorph Fuarium graminearum). Fungal Genet. Biol. 42:528-533.

44. Trail, F., Xu, H., Loranger, R., and Gadoury, D. 2002. Physiological and environmental aspects of ascospore discharge in Gibberella zeae (anamorph Fusarium graminearum). Mycologia 94:181-189.

45. Tschanz, A., Horst, K., and Nelson, P. E. 1975. Ecological aspects of ascospore discharge in Gibberella zeae. Phytopathology 65:597-599.

46. Vogelgsang, S., Widmer, F., Jenny, E., and Enkerli, J. 2009. Characterisation of novel Fusarium graminearum microsatellite markers in different Fusarium species from various countries. Eur. J. Plant Pathol. 123:477-482.

47. Zeller, K. A., Bowden, R. L., and Leslie, J. F. 2003. Diversity of epidemic populations of from small quadrats in Kansas and North Dakota. Phytopathology 93:874-880. 